\title{
Use of the automatic external defibrillator-pacemaker by ambulance personnel: the Stockport experience
}

\author{
A J GRAY, A D REDMOND, M A MARTIN
}

\begin{abstract}
In an attempt to reduce the number of people who die from a cardiac arrest in the Stockport area ambulances were equipped with automatic external defibrillator-pacemakers, and ambulance personnel were trained in their use. Over an 18 month period ambulance personnel attended 113 patients in cardiac arrest with these devices. One patient subsequently survived, and three patients survived for up to three days.

The reasons for these poor initial results include the failure of bystanders to provide cardiopulmonary resuscitation, a delay in calling for the ambulance, and too few defibrillators being available.
\end{abstract}

\section{Introduction}

In 1967 Pantridge and Geddes described a scheme in which mobile coronary care was given by doctors to patients who were in cardiac arrest in the community. ${ }^{1}$ Since then various schemes have been devised to resuscitate such patients outside hospital, the rationale being that survival depends on the rapid recognition and correction of arrhythmias, principally ventricular fibrillation, before the patient is transferred to hospital. In 1984 it was decided that a rapid response system was needed for patients who were having cardiac arrests outside hospital in the Stockport area. This area covers

Accident and Emergency Department, Stockport Infirmary, Stockport SK1 3UJ

A J GRAY, DRCOG, FRCS, senior registrar

A D REDMOND, MD, MRCP, consultant

Stepping Hill Hospital, Stockport

M A MARTIN, MD, MRCP, consultant cardiologist

Correspondence to: Mr Gray.
$126 \mathrm{~km}^{2}$, is predominantly urban with a population of 300000 people, and is covered by three ambulance stations. The central Stockport office was the station chosen for this project.

\section{Method}

The initial intention was to train selected ambulance personnel in the use of manual defibrillators to be used in the community but not to train them in intubation or drug administration. The regional health authority would not, however, sanction the use of manual defibrillators by ambulance personnel, and as a compromise the Heart-Aid Automatic External Defibrillator Pacemaker was used. During a pilot study only one device was used. Later five more devices were in use.

The ambulance personnel received eight hours of instruction in basic life support and in the use of the automatic external defibrillator and were subsequently examined. A protocol was established wherein the pulseless, unresponsive patient, after being given four "breaths" and five chest compressions, was connected to the defibrillator, and the machine administered three shocks if the patient was in ventricular fibrillation. If this was unsuccessful the patient was moved into the ambulance and the machine repeated the cycle after basic life support was repeated. If the patient did not respond basic life support was continued and the patient transported to the accident and emergency department. If the machine went into its pacing mode three full pacing cycles were permitted. If this did not produce a palpable femoral pulse and an obvious improvement in the patient's condition the patient was transferred to the ambulance, where the machine was reconnected. The machine was then allowed to proceed through a further three pacing cycles. If an output was obtained with the pacing the patient was left connected to the machine and transferred to the accident and emergency department (figure).

The Automatic External Defibrillator Pacemaker (AEDP Heart Aid, Cardiac Resuscitator Corporation, Oregon), has been described elsewhere. ${ }^{24}$ The machine is connected to the patient by two chest electrodes. The electrocardiographic recording is automatically analysed, and if the features of ventricular fibrillation are present the capacitors are charged and a DC shock is automatically delivered. If the ventricular rate is $25 /$ minute or less pacing begins. The electrocardiographic traces obtained were subsequently examined by a consultant cardiologist (MAM). 


\section{Results}

Over 18 months the ambulance personnel attended 113 patients in cardiac arrest with the automatic defibrillator. Sixty five $(57 \%)$ patients were in ventricular fibrillation when the ambulance personnel arrived, $29(26 \%)$ were in asystole, nine $(8 \%)$ were in an idioventricular rhythm, eight $(7 \%)$ in electromechanical dissociation, and two $(2 \%)$ in sinus bradycardia. Over the 18 months no patient survived long enough to leave hospital, although the details of one subsequent success are included. The mean time from the initiation of the 999 emergency call to the arrival of the ambulance was 4.5 minutes (range 1-9 minutes). Only $38 \%$ (43) of the patients who collapsed had received cardiopulmonary resuscitation from bystanders. This was often rudimentary, consisting only of chest compression or expired air respiration.

Six cases of cardiac arrest have been documented in patients in transit in vehicles that were equipped with the automatic defibrillator and manned by crews who had received advanced training. In all but one of these patients the initial arrhythmia was ventricular fibrillation (table I). Three patients survived long enough to be transferred from the accident and emergency department to intensive care units but subsequently died (table II) Immediately after the 18 month period a 63 year old man collapsed at home.

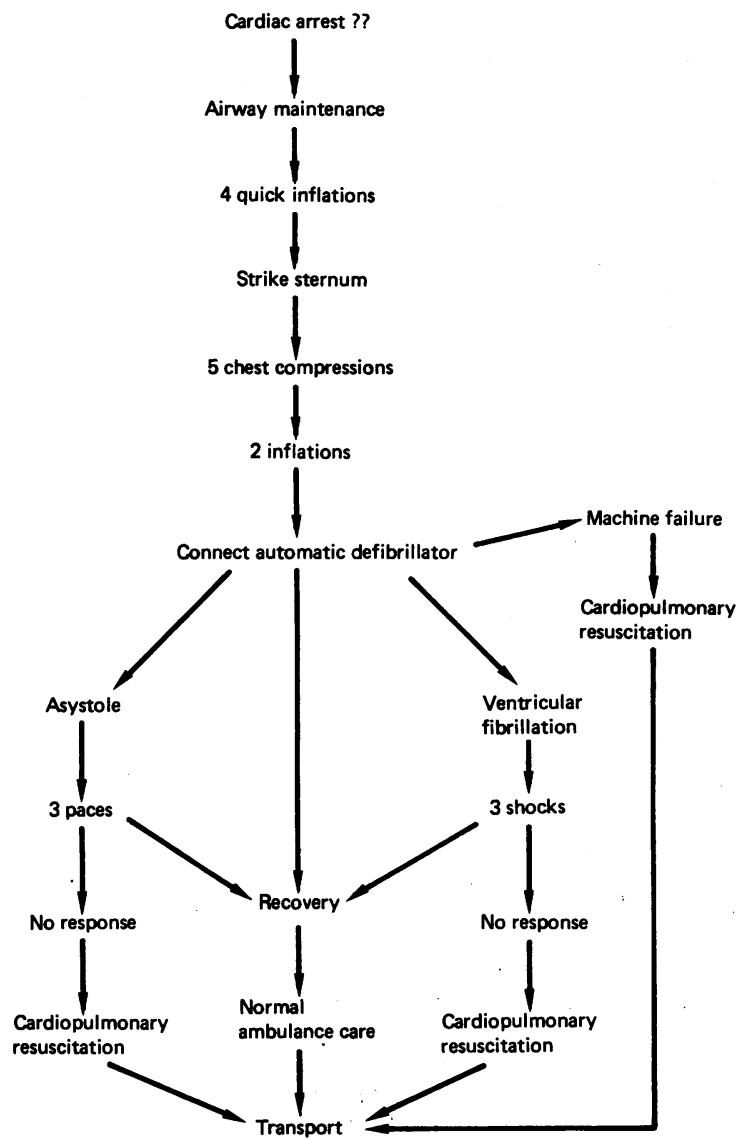

Protocol followed by ambulance personnel when using the automatic defibrillator.

TABLE I-Arrhythmias in patients who had a cardiac arrest on the way to hospital

\begin{tabular}{|c|c|c|c|c|}
\hline $\begin{array}{l}\text { Case } \\
\text { No }\end{array}$ & Initial arrhythmia & After defibrillation & On arrival & Outcome \\
\hline 1 & $\begin{array}{l}\text { Ventricular } \\
\text { fibrillation }\end{array}$ & Sinus bradycardia & Sinus bradycardia & $\begin{array}{l}\text { Died in emergency } \\
\text { department }\end{array}$ \\
\hline 2 & $\begin{array}{l}\text { Ventricular } \\
\text { fibrillation }\end{array}$ & Asystole & Asystole & $\begin{array}{l}\text { Died in emergency } \\
\text { department }\end{array}$ \\
\hline 3 & $\begin{array}{l}\text { Ventricular } \\
\text { fibrillation }\end{array}$ & $\begin{array}{l}\text { Idioventricular } \\
\text { rhythm }\end{array}$ & Sinus rhythm & $\begin{array}{l}\text { Died in emergency } \\
\text { department }\end{array}$ \\
\hline 4 & $\begin{array}{l}\text { Ventricular } \\
\text { fibrillation }\end{array}$ & $\begin{array}{l}\text { Ventricular } \\
\text { fibrillation }\end{array}$ & $\begin{array}{l}\text { Ventricular } \\
\text { fibrillation }\end{array}$ & $\begin{array}{l}\text { Died in emergency } \\
\text { department }\end{array}$ \\
\hline 5 & $\begin{array}{l}\text { Ventricular } \\
\text { fibrillation }\end{array}$ & Sinus rhythm & $\begin{array}{l}\text { Ventricular } \\
\text { fibrillation }\end{array}$ & $\begin{array}{l}\text { Died in emergency } \\
\text { department }\end{array}$ \\
\hline 6 & $\begin{array}{l}\text { Sinus bradycardia } \\
\text { (paced) }\end{array}$ & $\begin{array}{l}\text { Sinus bradycardia } \\
\text { (paced) }\end{array}$ & Asystole & $\begin{array}{l}\text { Died in emergency } \\
\text { department }\end{array}$ \\
\hline
\end{tabular}

TABLE II-Arrhythmias in patients who were transferred to intensive care

\begin{tabular}{|c|c|c|c|c|}
\hline $\begin{array}{c}\text { Case } \\
\text { No }\end{array}$ & $\begin{array}{c}\text { Initial } \\
\text { arrhythmia }\end{array}$ & $\begin{array}{c}\text { After } \\
\text { defibrillation }\end{array}$ & On arrival & Outcome \\
\hline 1 & $\begin{array}{l}\text { Ventricular } \\
\text { fibrillation }\end{array}$ & Bigeminal rhythm & Sinus rhythm & Died five hours later \\
\hline 2 & $\begin{array}{l}\text { Ventricular } \\
\text { fibrillation }\end{array}$ & Sinus bradycardia & Asystole & Died eight hours later \\
\hline 3 & $\begin{array}{l}\text { Ventricular } \\
\text { fibrillation }\end{array}$ & Sinus bradycardia & Sinus tachycardia & Died three days later \\
\hline
\end{tabular}

No cardiopulmonary resuscitation was performed by bystanders. The automatic defibrillator confirmed ventricular fibrillation, and the patient was shocked into asystole. After pacing, a sinus bradycardia developed with a cardiac output. This subsequently speeded up, and the patient was taken to hospital. He left hospital fit and well 10 days later.

\section{Discussion}

Cobb in Seattle devised a "saturation coverage" rapid response system using personnel from 36 fire stations who were trained in basic life support supplemented by advance life support from paramedical technicians who were trained in intubation, defibrillation, and the use of selected drugs. ${ }^{5}$ Eisenberg in King County, Washington, trained fire department and ambulance personnel as emergency medical technicians to deliver up to three $320 \mathrm{~J}$ shocks to patients in ventricular fibrillation in the community. ${ }^{6}$

In Brighton, Chamberlain and colleagues introduced mobile coronary care ambulances that were manned by selected ambulance personnel who were trained in defibrillation, the delivery of drugs, and intubation. But Hampton and Nicholas, in a randomised trial comparing the survival rates of patients who were transported to hospital in mobile coronary care units with the rates of those transported in ordinary ambulances, concluded that though mobile coronary care units could undoubtedly save a few lives, it was unlikely that they could contribute effectively to reducing mortality in the community from heart attacks. ${ }^{8}$ Hampton subsequently developed an extended programme of training for ambulance personnel, which included the use of defibrillators that were carried in the ambulances. The initial results were encouraging: ambulance staff were enthusiastic about the extended training, and the authors believed that the scheme could easily be established by other ambulance services in the United Kingdom. Our results compare unfavourably with other centres that have comparable schemes-that is, schemes in which personnel who have undergone training in the use of defibrillators do not intubate or use drugs at the scene of the cardiac arrest.

In the Nottingham series, of 72 patients who developed ventricular fibrillation in the community, 13 were discharged home. ${ }^{9}$ In Seattle, of 38 patients who were defibrillated, 10 left hospital. ${ }^{6}$ Stults defibrillated 12 patients successfully out of 64 who were in ventricular fibrillation in a rural community using trained ambulance technicians. ${ }^{10}$. The Brighton series, in which 27 people survived to leave hospital out of 160 who had been defibrillated in the community, is not really comparable as many of these patients were intubated and received drugs at the scene of the cardiac arrest. ${ }^{7}$

The reasons for our poor results are not clear. Our response times from the time that the 999 emergency call was received to the arrival of the ambulance at the scene compare favourably with those in other series. We could not document accurately, however, the time from the patient's collapse to the initiation of the 999 emergency call. Obviously, this period of time is crucial if early defibrillation is to be achieved. The proportion of patients in our series who were in ventricular fibrillation and asystole on presentation is broadly similar to those of other studies: Eisenberg $57 \%$ in ventricular fibrillation, $28 \%$ in asystole ${ }^{6}$; Briggs $77 \%$ in ventricular fibrillation, $22 \%$ in asystole. ${ }^{7}$ Nothing suggested that a higher percentage of patients presented to us with unfavourable arrhythmias, such as asystole. In our series of 113 patients only six had a cardiac arrest in transit. It is perhaps important that of the 13 survivors of the Nottingham series, seven were from the group that suffered arrests 
in transit in the ambulance, which emphasises the need for early defibrillation to produce a favourable outcome.

Proportionally fewer of our patients received cardiopulmonary resuscitation from bystanders compared with patients in other series. Cobb noted that the hospital mortality in resuscitated patients was halved if a bystander initiated resuscitation within four minutes of the arrest. ${ }^{5}$ This has been confirmed by other workers.

The one variable between our series and the others is the use of the automatic external defibrillator. Jaggarao from Brighton described the use of an automatic defibrillator in a small group of patients. ${ }^{3}$ Five patients survived out of 11 who were defibrillated in the community. Several patients were intubated, however, before the defibrillator was used, and intravenous or intracardiac drugs were administered to some patients. Cummins, in a field evaluation of an automatic defibrillator reported a sensitivity (defined as whether patients in ventricular fibrillation received a counter shock) of $81 \%$ and a specificity (defined as the rate at which the device successfully recognised and did not shock non-ventricular fibril-

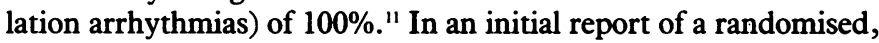
controlled comparison of automatic defibrillators and manual defibrillators, each with the same emergency medical technicians, the sensitivities and specificities of the two systems were not appreciably different. Indeed, the only major difference was that the time interval from "power on" of the defibrillator to delivery of the first countershock was shorter with the automatic device than with the manual device ( 1.07 minutes compared with 1.57 minutes). Cummins suggested that though he did not yet have sufficient numbers of patients to reach firm statistical conclusions, the performance of automatic external defibrillators was comparable to that of manual defibrillators. We had one case where the electrocardiogram of a patient who collapsed in the street was interpreted by the automatic defibrillator as ventricular fibrillation, and the patient was defibrillated. Subsequent inspection of the trace, however, indicated that the rhythm was a low voltage sinus rhythm. The patient had in fact collapsed from a ruptured abdominal aortic aneurysm. Almost without exception analysis of the tape records showed that the machine functioned as it was designed to.

An inspection of the rhythm tapes from the defibrillators suggests that patients in ventricular fibrillation who were being shocked into potentially viable rhythms "decayed" when being transferred to the accident and emergency department, usually to asystole or back to ventricular fibrillation despite ventilation with bag and mask and $100 \%$ oxygen and external cardiac massage where necessary. If advanced life support were provided to such patients by the ambulance personnel more "saves" might occur. Good basic life support is almost impossible in a moving vehicle. Pacing was unsuccessful in most of the patients who presented with asystole as the initial arrhythmia. One patient was paced into ventricular fibrillation. Pacing, however, was often successful in patients who were defibrillated into asystole, conversion being either back to ventricular fibrillation or into a sinus bradycardia or idioventricular rhythm.

Some of these arrhythmias were accompanied by a cardiac output that had disappeared by the time the patients arrived at the accident and emergency department. One criticism that might be levelled at our protocol is that if the initial automatic sequence of identification of the rhythm, charge of the capacitors, and delivery of the shock is unsuccessful the patient is then transferred to the vehicle and the sequence is repeated. Furthermore, if the journey to the accident and emergency department takes more than five minutes the vehicle is stopped and the "heart aid" cycle is repeated. Perhaps patients would benefit from a rapid transfer to the accident and emergency department rather than be delayed with a second, usually unsuccessful, phase with the automatic defibrillator.

What has been achieved at the end of the 18 months in Stockport? The ambulance personnel have received extended training, which included the use of defibrillators, and most are keen to progress to the use of manual defibrillators, which has now been sanctioned by the regional health authority. Our resulis indicate that successful resuscitation of patients in cardiac arrest outside hospital will be achieved only if defibrillators are provided in ambulances in sufficient numbers and if this is accompanied by a public education programme. The public lacks awareness in the management of a cardiac arrest, and it will be interesting to see our figures after the Save a Life Campaign. Our aim is to deploy more defibrillators to achieve a near "saturation" coverage. We estimate that a further 14 will be required. Hampton and his colleagues rightly say that there is a need for defibrillators in ambulances; but providing defibrillators is not enough.

We thank Mr Graham Underhill, superintendent, Stockport District Ambulances, for his support in this project.

\section{References}

1 Pantridge JF, Geddes JS. A mobile intensive care unit in the management of myocardial infarction. Lancet 1967; ii:271-3.

2 Diack AW, Welborn WS, Rullman RG, Walter CW, Wayne MA. An automatic cardiac resuscitator for emergency treatment of cardiac arrest. Med Instrum 1974;13:78-81.

3 Jaggarao NSV, Heber M, Grainger R, Vincent R, Chamberlain DA, Aromson AL. Use of an automated external defibrillator-pacemaker by ambulance staff. Lancet 1982;ii:73-5.

4 Heber M. Out-of-hospital cardiac arrest using the "heart-aid", an automated external defibrillator-pacemaker. In $f$ Cardiol 1983;3:456-8.

5 Cobb LA, Baum RS, Alvarex H III, Schaffer WA. Resuscitation from out of hospital ventricular fibrillation: 4 years' follow-up. Circulation 1975;51, 52 (suppl III):223-8.

6 Eisenberg MS, Copass MK, Hallstrom AP. Treatment of out-of-hospital cardiac arrest with rapid defibrillation by emergency medicine technicians. N Engl f Med 1980;302:1379-83.

7 Briggs RS, Brown PM, Crabb ME, et al. The Brighton resuscitation ambulances: a continuing experiment in pre-hospital care by ambulance staff. $\mathrm{Br} M e d$ F 1976;ii:1161-5.

8 Hampton JR, Nicholas C. Randomised trial of a mobile coronary care unit for emergency calls. BrMed f 1978;i:1118-21.

9 Rowley JM, Garner C, Handy M, Hampton JR. Simple training programme for ambulance personnel in the management of cardiac arrest in the community. Br Med f 1985;291:1099-101. 10 Stults KR, Brown DD, Schug VL, Bean JA. Prehospital defibrillation by emergency medical technicians in rural communities. N Engl f Med 1984;310:219-23.

11 Cummins RO, Eisenberg MS, Graves JR et al. Automatic external defibrillators used by emergency medical technicians: a controlled clinical trial. Crit Care Med 1985;13:945-6.

(Accepted 28 January 1987)

Is metformin contraindicated in a man of 64 with maturity onset diabetes who has had an uncomplicated myocardial infarction?

Metformin, a biguanide effective in managing maturity onset diabetes, may have an additive effect when given with the sulphonylureas. Metformin is similar to its predecessor phenformin, which it structurally resembles, but the risks of lactic acidosis are much less prominent and side effects are not usually a problem. Studies of phenformin-now withdrawn-showed that it might have a deleterious effect on cardiovascular mortality, possibly by increasing the blood pressure and the heart rate. A five year study in patients who had taken phenformin after myocardial infarction, however, showed no increase in mortality. There is no evidence that metformin compromises the cardiovascular system, and in patients with uncomplicated myocardial infarction in whom there is no overt risk of congestive cardiac failure the risks of using metformin must be small. If the patient should develop heart failure the risk of lactic acidosis would increase and the drug should be withdrawn.-L M SHAPIRO, senior registrar in cardiology, London.

What is the incidence of Down's syndrome in the United Kingdom and has it changed in recent years?

The incidence of Down's syndrome in the United Kingdom is somewhere between 1.09 per thousand live births (Liverpool area) $)^{1}$ and 1.2 per thousand (Scotland). These figures are lower than they were in the era before amniocentesis, and the fall might be attributed either to the prenatal screening of women older than 35 or to a reduction in the number of live births in women aged over 35. Ferguson-Smith (Scottish data) thought that the prenatal screening programme had reduced Down's syndrome by between $5 \cdot 8 \%$ and $8 \cdot 8 \%$, a reduction that he judged as disappointing. ${ }^{2}$ In Liverpool the mean age of women giving birth to infants with Down's syndrome fell from 36.7 years in 1961 to 29 years in 1979, and this fall in the number of pregnancies in older mothers was considered sufficient to explain the falling incidence. In the United Kingdom only half of mothers over the age of 36 were being screened for Down's syndrome. In the Scottish study $7 \%$ of women declined prenatal diagnosis on religious or moral grounds and $16 \%$ were seen too late. The rest were not offered the test.—M BARAITSER, consultant in clinical genetics, London.

1 Owens JR, Harris F, Walker S, McAllister E, West L. The incidence of Down's syndrome over a 19 year period with special reference to maternal age. $\mathcal{F}$ Med Genet 1983;20:90-3.

2 Ferguson-Smith MA. Prenatal chromosome analysis and its impact on the birth incidence of chromosome disorders. Br Med Bull 1983;39:355-64. 